MS20-P09

\section{Results and experience using recycled beam at the CXI instrument of LCLS}

\author{
Mark Hunter ${ }^{1}$ \\ 1. LCLS at SLAC National Accelerator Laboratory, Menlo Park, \\ United States of America \\ email: mhunter2@slac.stanford.edu
}

Beam time at X-ray Free Electron Lasers (FELs), such as the Linac Coherent Light Source (LCLS), is currently oversubscribed several fold. At LCLS, multiplexing has been used to increase access by using a monochromator to extract a 0.5 or $1 \mathrm{eV}$ slice of the pink beam bandwidth $(\sim 50$ $\mathrm{eV})$ and deliver it to a different experiment; this setup is commonly used for multiplexing of the monochromatized beam at X-ray Pump Probe (XPP) and Coherent X-ray Imaging (CXI) instruments. However, many experiments at CXI utilize a small fraction $(<50 \%)$ of the incident beam due to a combination of small scattering cross sections and $<50 \mu \mathrm{m}$ path lengths. Therefore a new chamber and re-focusing optics were designed to make use of the transmitted beam from a primary experiment at CXI. The Serial Sample Chamber (SSC) was commissioned in July of 2016 and started parasitic operations immediately, contributing to single-wavelength anomalous difference (SAD) protein crystallography experiments conducted the same month. Since its successful commissioning, in-house experiments and user experiments have been conducted in SSC, expanding the amount of available beam time to the community. Starting in 2017, SSC became the dedicated place for the Protein Crystallography Screening (PCS) program at LCLS. Results from using SSC for experiments and the quality of the recycled beam will be discussed including discussions of the challenges facing the parasitic experimental setup. The serial data collection setup will soon be implemented at European XFEL at the SPB/SFX instruments.

Keywords: Crystallography, X-ray FEL
MS20-P10

\section{Interaction of non-ideal, multicomponent solid solutions with water: a simple algorithm to estimate final equilibrium states}

Rosa María Rodríguez Galán ${ }^{1}$, Manuel Prieto ${ }^{1}$

1. Department of Geology. University of Oviedo, Oviedo, Spain email: rosa@geol.uniovi.es

Interaction of minerals with water frequently yields dissolution-coprecipitation processes in which foreign ions incorporate into the solid structure substituting for the major ion. By this way, coprecipitation often controls the transport and fate of harmful ions in the environment and can be exploited for environmental remediation.

Geochemical modeling and experimental studies of these aqueous - solid solution (AQ SS) processes are typically performed using pure minerals. However, the host minerals could be binary solid solutions and the study of their interaction with a third dissolved ion would require considering ternary solid solutions (TSS). The first step in modeling coprecipitation processes is providing the equilibrium state to which a specific AQ-SS system will tend to react. However, computer codes like PHREEQC does not deal with non-ideal ternary or higher order solid solutions and GEM-Selektor [Wagner et al., 2012; Kulik et al., 2013] is the only modeling code that allows calculating equilibrium in AQ-SS systems that involve non-ideal multicomponent solid solutions. We have implemented a simple algorithm (AQ-TSS) to work in a PHREEQC context via the BASIC interpreter tool of this modeling code. AQ-TSS estimate equilibrium in AQ-SS systems involving non-ideal ternary solid solutions (regular, subregular, or any-type). The $(\mathrm{Pb}, \mathrm{Sr}, \mathrm{Ba}) \mathrm{SO}_{4}$ solid solution is used as example system. The three binary joints are considered separately. Non-regular solid solutions are treated by combining a set of regular models defined for specific ranges of composition and neglecting ternary interactions, which represent a minor contribution when two of the three components occur in minor amounts. Such a scenario is realistic for ambient temperature processes in which the host solid solutions exhibit wide miscibility gaps and the dissolved metal occurs in trace or minor amounts. The algorithm has been tested by comparing the results obtained by AQ-TSS with those obtained using PHREEQC with the same binary non-ideal solid solutions and by reproducing some calculations performed by Vinograd et al. (2013) using GEMS (Kulik et al., 2013). The results obtained by AQ-TSS and PHREEQC are virtually equal. The results obtained using GEMS are of the same order of magnitude, the difference $(\sim 5 \%)$ being reasonable given the different basis involved in both algorithms.

The extension to non-ideal solids solutions of higher order is feasible, but the formulations become bulkier with each added component. The lack of rigorous experimental data involving aqueous-ternary solid solution systems is a major obstacle and a challenge for future research. 